\title{
Current possibilities of cervical precancerous lesions screening in Slovakia: prevalence of high risk human papillomavirus in patients with cytological diagnoses of atypical squamous cells of unknown significance
}

\author{
Kolnikova $\mathrm{G}^{1}$, Ondrusova $\mathrm{M}^{2,3}$, Repiska $\mathrm{V}^{4}$, Drobna $\mathrm{R}^{5}$, Marinova $\mathrm{P}^{1}$, Meciarova $\mathrm{I}^{1}$, \\ Rampalova $\mathbf{J}^{1}$, Ondrias $\mathrm{F}^{1}$
}

Alpha Medical Patologia Ltd., Bratislava, Slovakia. mudr.kolnikova@gmail.com

\begin{abstract}
Introduction: It has been confirmed, that there is a causal relationship between persistent infection of high risk human papillomavirus (HR-HPV) and the development of cervical cancer. In population of women older than 30 years HPV infection becomes a significant etiological factor of precancerous lesion of the cervix, but HPV infection may spontaneously regress in the majority of the cases.

Patients and methods: The analysed study group consisted of 397 samples with cytological diagnosis of atypical squamous cells of unknown significance (ASCUS). All cases underwent HPV DNA testing using the Hybrid Capture 2 (HC2) assay. We analysed prevalence of HR-HPV and a viral load expressed as relative light units/ cut off ratio (RLU/CO) in different age groups with cytological diagnoses of ASCUS.

Results: The prevalence of HR-HPV with cytological diagnoses of ASCUS was detected in $44 \%$. The prevalence of HR-HPV between patients aged 17-29 and between patients aged 30-40 was $55 \%$ and $48 \%$ respectively and we detected significant reduction of prevalence $(28 \%)$ in patients older than 41 years.

Conclusion: Based on the results of presented study we assumed that age the 40 and over is crucial for the development of serious precancerous lesions in Slovakia, thus this age group is the most suitable for HPV triage of ASCUS. As a refinement of that type of ASCUS triage we recommend to add to the algorithm quantitative measurement of viral load in the specimens in the form of RLU/CO ratio (Fig. 3, Ref. 27). Text in PDF www.elis.sk. Key words: cervical cancer, ASCUS, management, HPV detection methods, clinical validation.
\end{abstract}

Cervical cancer is estimated to be the third most common cancer and the fourth most common cause of cancer deaths worldwide in women. Long-time incidence and mortality trends in Slovakia are similar to developing countries (1). Although Slovakia is ranked among the economically more advanced countries, up to now it has not succeeded in reducing mortality of cervical cancer due to the lack of interest of women in screening for the disease and the opportunistic character of its organisation. In the last published year 2007 (2), the occurrence of cervical cancer in Slovakia represented 627 cases (age-adjusted incidence to the World standard ratio (ASR-W) was 16.6/100 000) and 237 deaths from cervical cancer (ASR-W mortality was 5.7/100 000). Analysis of the agespecific incidence rates in y. 2003-2006 show, that cervical cancer did not occur before the age of 20 . It started to increase after the age 25 with the highest incidence in the age group of 45-49 years

${ }^{1}$ Alpha Medical Patologia Ltd., Bratislava, Slovakia, ${ }^{2}$ St. Elizabeth Univer-
sity of Health and Social Sciences, Bratislava, Slovakia, ${ }^{3}$ Cancer Research
Institute, Slovak Academy of Sciences, Bratislava, Slovakia, ${ }^{4}$ Institute of
Medical Biology, Genetics and Clinical Genetics, Faculty of Medicine,
Comenius University in Bratislava, Bratislava, Slovakia, and ${ }^{5}$ Central la-
boratories - west, Alpha Medical co., Bratislava, Slovakia Address for correspondence: G. Kolnikova, MD, Alpha Medical Patologia Ltd., Ruzinovska 6, SK-826 06 Bratislava, Slovakia.
(1). Persistent infection with HR-HPV is a necessary step in the development of cervical cancer and other anogenital carcinomas $(3,4)$. Infection with HR- HPV has also been etiologically linked with head and neck squamous cell carcinoma (5). The majority of HPV infections of the cervix are transient and undergo clearance or become latent within 1-2 years of detection, especially among younger women, while in population of women older than 30 it becomes a significant etiological factor of precancerous lesion of the cervix $(6,7)$. Incorporation of HR-HPV detection into cervical screening programs besides conventional cytological screening may improve the ability to identify women at risk of cervical cancer (8). According to our bibliographic research, there are only few studies about the prevalence of sexually transmitted infection in Slovakia (e.g. urogenital chlamydial infection in women from eastern Slovakia) (9). Our study is the first analysis of prevalence of HR-HPV of patients with cytological diagnosis of ASCUS and also the first attempt to introduce the viral load of HPV in the specimen in the management guidelines of precancerous lesions in Slovakia.

\section{Patients and methods}

In the reporting period of 36 months we analysed 397 cervical cytology samples for the presence of HR-HPV. The age of patients 
469-473

was between 17 to 71 years $(n=397)$, median age 32 years. Each patient had a cytological diagnosis of ASCUS diagnosed in our laboratory. None of the patients was vaccinated, duplicate examinations were excluded from the study group. All cases underwent HPV DNA testing using the commercially available Hybrid Capture 2 assay by Quiagene/Digene. Each sample was examined for the following high-risk types of HPV: 16, 18, 31, 33, 35, 39, 45, $51,52,56,58,59,68$. During the sampling, transport, calculation of luminescence and interpretation of results we followed the instructions of the manufacturer. We used the original (Dynex Slovakia, Ltd.) diagnostic-kit. The Digene HC2 High-Risk HPV DNA Test was the first clinically validated HPV test on the market. The test is an in vitro nucleic hybridisation assay using an RNA probe mixture from 13 high risk (HR) HPV types, without distinguishing between them. The assay utilizes signal amplification technology: the semiquantitative viral load of a positive sample is indicated by a chemiluminescent signal measured in relative light units (RLU) of the sample to that of the cut-off (CO) value of the positive control. The manufacturer established an RLU ratio of 1.0 or higher as positive. The exact RLU values are not routinely reported as the test is used in a qualitative fashion (positive vs. negative). In our study group positivity was further divided to borderline positivity $(1 \leq \mathrm{RLU} / \mathrm{CO} \leq 2)$, weak positivity $(2.0<$ $\mathrm{RLU} / \mathrm{CO} \leq 10.0)$, mild positivity $(10.0<\mathrm{RLU} / \mathrm{CO} \leq 100.0)$ and high positivity $(100.0<\mathrm{RLU} / \mathrm{CO})$.

\section{Results}

The number of analysed samples with cytological diagnosis of ASCUS was $n=397$. Age categories: The total number of the analysed samples of patients up to 29 years of age was $128(32 \%)$, between the age of 30 to 40 it was $141(36 \%)$ and for ages of more than 40 it was 128 (32\%). From the total number of 397 patients negativity for HR HPV was detected in 221 cases (56\%), positivity was in 176 cases (44\%). We detected negativity for HR HPV in ASCUS patients in 54 samples up to the age of 29, 71 samples between the age of 30 to 40 and 96 samples in patients up to 41 years of age. Positivity for HR HPV was detected in 71 patients (55\%) up to 29 years old, in 69 patients (48\%) between 30 and 40 years of age and in 36 patients $(28 \%)$ older than 41 years (Fig. 1). During more detailed analysis of RLU/CO results, we detected

$\square$ HR HPV+

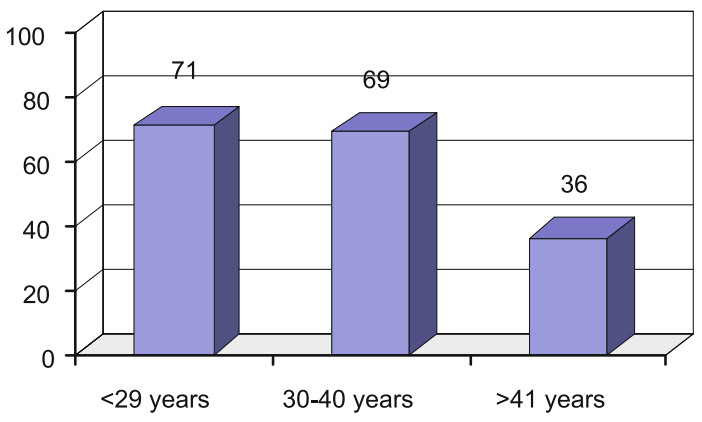

Fig. 1. Age distribution of ASCUS patients with HR HPV positivity

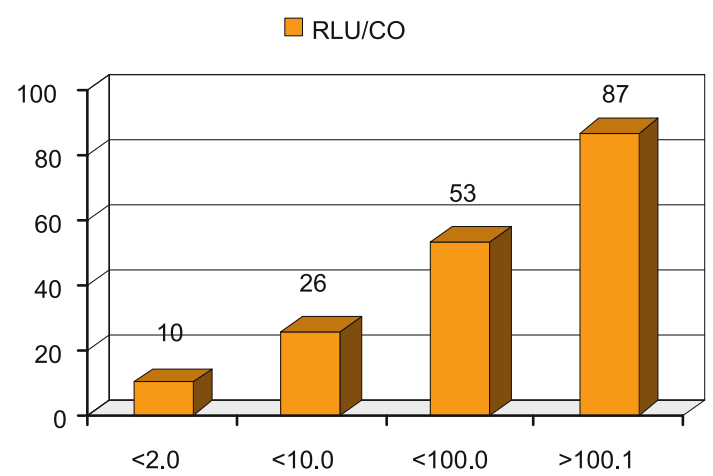

Fig. 2. Number of patients and the degree of RLU/CO value at HR HPV positivity.

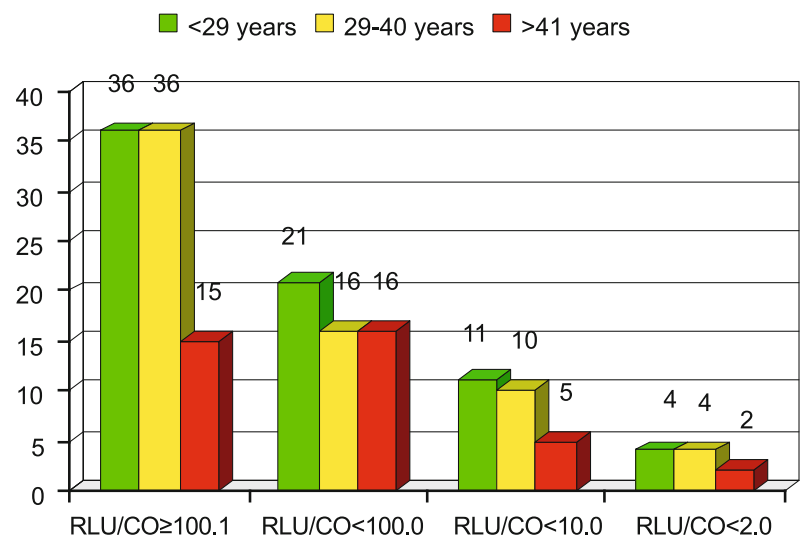

Fig. 3. The number and the value of RLU/CO in HR HPV positive ASCUS patients of different age groups.

high positivity in samples of 87 patients, mild positivity in samples of 53 patients, weak positivity in samples of 26 patients and borderline positivity in samples of 10 patients (Fig. 2). Figure 3 shows the number and the RLU/CO value of HR HPV positive ASCUS patients of different age groups. The number of patients with strong positivity for HR HPV before the ages of 29 and 40 years was the same, 36 , the highest of all age groups.

\section{Discussion}

ASCUS is the most common abnormal cytological diagnoses within cervical cancer screening $(10,11)$. The risk of invasive cancer in patients with ASCUS cytology is low, ranging from 0.1 $\%$ to $0.2 \%$ according to the literature $(12,13)$.

Emerson et al (13) analysed 15860 patients with cervical cytology a period of 9 years. Patients with previous dysplasia or carcinoma were excluded from analysis. A diagnosis of ASCUS was made in 1117 cases $(7.0 \%)$. After excluding 345 patients with previous dysplasia or human papillomavirus-related diagnoses and 129 patients with no follow-up specimens, 643 study patients remained. Squamous intraepithelial lesion (SIL) or dysplasia was subsequently diagnosed in 197 patients (30.6\%). High-grade squamous intraepithelial lesion (HSIL) or at least moderate dysplasia was diagnosed in 64 patients (10.0\%). In 21 cases (3.3\%) the high-grade dysplasia 
developed more than 2 year after the first ASCUS diagnosis. Follow-up disclosed no cases of invasive carcinoma. Among ASCUS patients followed for up to 9 year, $20 \%$ develop only low-grade SIL or mild dysplasia and $10 \%$ develop HSIL or moderate or severe dysplasia. In $3.3 \%$ of patients HGSIL was found out within 2 years from ASCUS diagnosis, after 9 years of observation SIL or dysplasia occurred in $30.6 \%$ of cases, $10 \%$ out of which was HGSIL.

ASCUS-LSIL Triage Study Group (ALTS) pillar study (10) was undertaken to compare alternative strategies for the initial management of a cytological diagnosis of ASCUS. A total of 3488 women with a community-based ASCUS interpretation were randomly assigned to immediate colposcopy, triage that was based on HPV DNA testing or conservative management based on repeat cytology. Prevalence of HR-HPV in ASCUS cytological diagnosis was $45 \%$. The study end point was 2 -year cumulative diagnosis of CIN grade 3. To compare triage tests, Solomon et al in ALTS (10) re-estimated the performance of HPV and cytology in successfully referring women with underlying CIN grade 3. A single enrolment HPV test identified $92.4 \%$ of the women diagnosed with CIN grade 3. Serial cytology, even at an ASCUS threshold, would have required two visits to achieve similar sensitivity (95.4\%) and would have referred $67.1 \%$ to colposcopy.

Within the category of women of less than 30 years of age the HPV infections can reach prevalence of $50 \%$ which is a lot more than originally assumed (14). Even the incidence and prevalence of HPV infections between postmenopausal women increases (15). Fortunately, the majority $(80-90 \%)$ of HPV infections are only of transient nature and they spontaneously disappear after several months leaving no morphological changes. During this transitional period we can observe only mild symptoms of HPV infection at the epithelium of the cervix (in the form of LG-SIL or CIN I) or the infection occurs not only clinically but also morphologically silently, i.e. without cytological abnormalities (14). Only persistently infected cervical cells have a substantial likelihood of gradually growing into diagnosable HSIL (16). This means that the fact of HPV presence (even the HR HPV) is not sufficiently serious and important information. It turned out that a viral "load", i.e. number of copies of genome of high-risk type of HPV, plays an important role in the emergence and development of precancerous lesions of the cervix, beside the genotypes of $\operatorname{HPV}(14,17)$. Several overview studies have shown that women with normal cytology but increased "load" of HR-HPV have an increased risk of developing serious CIN lesions. In patients with already developed CIN lesion and detected smaller amount (less than $10^{4}$ copies per sample) of HR-HPV types, the CIN lesion regressed. At a higher viral load the progression of CIN lesion to more serious lesions has been observed more frequently (17). Recent randomized controlled cervical screening trials have shown that HR-HPV testing on cervical scrapes can be also used as a primary tool for cervical cancer screening, given its superior sensitivity and negative predictive value for cervical pre-cancer compared with cytology (17). To make a distinction between HPV test performances for outcome measure "(pre)malignant disease" vs outcome measure "HPV presence" the terms "clinical sensitivity and specificity" and "analytical sensitivity and specificity" were introduced $(17,18,19$,
20). Many different HPV tests exist nowadays, which all have their technical, analytical and clinical properties. The choice for an HPV test for a given purpose should therefore not solely be based on practical considerations, but also on the intention of its use. If we e.g. expect detection of epidemiological studies of HPV incidence within certain region or an age group of population, methods with high analytical sensitivity will be more appropriate. These are able to detect very low amounts of HR-HPV (even less than 10 copies of viral DNA per sample) regardless of whether it has or has not any prognostic significance for a patient. Their disadvantage among others is their high susceptibility to contamination and, as a result of that, development of false positive results $(17,18,19,20)$.

However, in case of a patient with borderline ASCUS cytology to detect if there is a potentially prognostically significant amount of HR HPV in her lesion, it is recommended to use a method with high clinical sensitivity. High clinical sensitivity means that we detect clinically significant amount of HR-HPV, i.e. the amount that is capable of inducing development and progression of disease within the sense of precancerous lesion. The appropriate methods for such purposes are methods with sensitivity of 1pg HPV (approximately 50000 copies per $1 \mathrm{ml}$ sample). Methods with analytically lower sensitivity do not detect clinically insignificant amounts of HR HPV, e.g. "HPV presence", they detect clinically relevant HPV infections causing “(pre)malignant disease” $(17,18,19,20)$.

The Digene Hybrid Capture 2 High-Risk HPV DNA Test (HC2), was the first FDA (Food and Drug Administration) approved clinically validated HPV test on the market $(10,20,21)$.

Saefian et al (22) compared development of dysplasia between HPV-negative and HPV-positive ASCUS patients. Overall only 22 of 1559 (1.4\%) HPV-negative ASCUS women developed CIN grade 3 or worse during follow-up compared with 269 of 1767 (15.2\%) HPV-positive ASCUS women ( $<0.001)$. HR HPV was also detected by the hybrid capture 2 method (HC2). The specificity of HPV testing ( $84 \%$ ) was significantly higher than cytology using an ASCUS threshold $(71 \%)(p<0.001)$. Co-testing with cytology and HPV testing at 12 months resulted in even lower specificity (61\%). The positive predictive value for CIN3 or worse was low for cytology (2.6 \%), Hybrid Capture 2 (3.8\%), and co-testing with cytology and HPV testing $(2.2 \%)$. The negative predictive value for all three management strategies was very high $(99.70$ $\%, 99.82 \%$, and $100.0 \%$ for HPV testing, cytology, or co-testing, respectively). The results show that the "HPV negative" patients with ASCUS cytological diagnosis should return to routine screening because the probability of the CIN2-CIN3 lesion formation is very low. However, if increased surveillance is chosen, a single HPV test for carcinogenic types at 12 months has significantly higher specificity and lower referrals than cytology.

Clinically validated HR HPV DNA test in combination with negative cytological examination has negative predictive value of $99-100 \%$ for the development of CIN 2 - CIN $3(10,16,23)$.

Based on described and several other recent studies, the American Society for Colposcopy and Cervical Pathology (ASCCP) (24), updated the consensus screening guidelines and the management of abnormal cervical cancer screening tests and cancer precursor. For women "of general population" with ASCUS cytology reflex 
HPV testing is preferred. For women with HPV negative ASCUS, whether from reflex HPV testing or co-testing, repeat co-testing at 3 years is recommended. For women with HPV positive ASCUS, wheter from reflex HPV testing or co-testing, colposcopy is recommended. When colposcopy does not identify CIN in women with HPV positive ASCUS, co-testing at 12 month is recommended (not less than 12 months). For women with ASCUS cytology and no HPV result, repeat cytology at 1 year is acceptable. If the result is ASCUS or worse colposcopy is recommended, if the result is negative, return to cytology testing at 3 years interval is recommended. Endocervical sampling is preferred for women in whom no lesions are identified and for those with an inadequate colposcopy, but is acceptable for women with an adequate colposcopy and a lesion identified in the transformation zone. Because of the potential of overtreatment, the routine use of diagnostic excisional procedures such as loop electrosurgical excision for woman is unacceptable. There are different management guidelines for women aged 21-24 years, pregnant women, postmenopausal women and women aged 65 years considering exit from screening. For women aged 21-24 years with ASCUS, cytology alone at 12 month is preferred, but reflex HPV is acceptable. If HPV testing is negative, return for routine screening is recommended (cytology alone). If HPV is positive repeat cytology is recommended. Immediate colposcopy or repeat HPV testing is not recommended. Management guidelines for pregnant women with ASCUS are identical to those described guidelines for "general population". Endocervical curettage is unacceptable. For pregnant women, who have no suspected CIN 2+ abnormality, postpartum follow up is recommended. Postmenopausal women with ASCUS should be managed in the same manner as women in the general population, except when considering exit from screening, when HPV negative ASCUS result should be considered as abnormal. Consensus guidelines is recommended repeat screening in year (co-testing preferred, but cytology is acceptable) (24).

European guidelines for quality assurance in cervical screening (2nd edition) (25) for the management of ASCUS are slightly different. For management for ASCUS three alternatives are recommended: HR HPV DNA testing, repeat cytology and referral for colposcopy. Reflex HR HPV testing is the preferred option when liquid based cytology is used. HPV positive cases should be referred for colposcopic evaluation. HPV testing can be repeated after 12 months when no CIN is found on colposcopy and biopsy. HPV negative women should be recommended repeat cytology after one year. A second acceptable option is repeat cytology. If it is negative, return to routine screening is recommended. If the first repeat cytology is again ASCUS, it should be referred for colposcopy. If any of the follow up smears is greater than ASCUS then referral colposcopy is advised. Referral for immediate colposcopy is another alternative, which many experts consider to be overmanagement. European guidelines recommended that clinicians should be guided by their own National guidelines (25).

In presented study group $56 \%$ of samples of patients with ASCUS were negative for HR HPV. The HR HPV prevalence in our group with ASCUS cytology diagnoses was $44 \%$, which is in accordance with international literature $(10,21,26)$. A significant decrease of positivity for HR HPV was detected after the age of 40 years $(28 \%)$. We assumed that this age will be crucial for the development of precancerous lesions within the Slovak population. Based on the results it can be assumed, that HPV infection in women of around 40 years of age is more likely to persist and precancerous lesion occurs. The hypothesis was indirectly confirmed also by the fact, that development of cervical cancer lasts approximately $10-12$ years and the highest age specific incidence of cervical cancer in Slovakia is in $45-50$ years of age (1).

The patients were classified also on the base of the intensity of positivity of the RLU/CO value. Our group showed significant positivity for HR HPV (above 100.1) recorded in $49 \%$ for age group up to 40 years of age, in the age group of older than 41 years of age it was $17 \%$ of patients. There are several studies analysed correlation between HC2 HR-HPV DNA test chemiluminescence intensity from cervical samples with follow-up histologic results. Jarboe et al (27) tested the hypothesis that HR-HPV positives with low positive RLU/CO ratios (1-10) had a lower prevalence of cervical intraepithelial neoplasia $2-3$ on histologic follow up. RLU/ CO ratios for 388 consecutive HR-HPV positive cervical cytological specimens interpreted as ASCUS were reviewed. Individual RLU/CO ratios were compared with outcome histologic diagnosis in cases with colposcopic follow-up and tissue sampling (biopsy and/or endocervical curettage; $n=236$ ). An RLU/CO ratio of 10 or less was associated with a significantly lower prevalence of CIN2/ CIN3 on biopsy outcome after result of ASCUS. Origoni et al (9) analysed a correlation between viral load, expressed as RLU/CO, and the severity of cervical lesions existed in 614 ASCUS cases. $\mathrm{HC} 2 \mathrm{RLU} / \mathrm{CO}$ values, categorised into five classes, were correlated to clinical outcomes and statistically analysed. A significant correlation ( $p<0.0001)$ was observed between increasing RLU values and the prevalence of high-grade CIN (CIN2/CIN3). The mean RLU/CO values were for negative cases 68.1 , for low-grade 172.5 and for high-grade lesions 1020.0. CIN2/CIN3 ranged from $4 \%$ for $0<\mathrm{RLU} / \mathrm{CO} \leq 1$, to $5 \%$ for $1<\mathrm{RLU} / \mathrm{CO} \leq 10$, to $9 \%$ for $10<\mathrm{RLU} / \mathrm{CO} \leq 100$, to $23 \%$ for $100<\mathrm{RLU} / \mathrm{CO} \leq 1000$ and to $48 \%$ when RLU/CO were $>1000(\mathrm{p}<0.05)$. The HPV viral load in ASCUS cases significantly correlates with the severity of cervical cancer precursors. These data may have prognostic value, as they significantly correlate with the probability of a CIN2+ (11).

The number of patients with strong positivity for HR-HPV before the ages of 29 and 40 years the highest of all age groups, but we must not forget the fact, that the majority of HPV infections are transient and undergo clearance or become latent within 1-2 years.

In conclusion, 2012 American updated consensus guidelines of ASCCP for the management of women with ASCUS on cytology preferred reflex HPV DNA testing. HPV testing results determine the follow-up management of women, depending on the age. European guidelines are slightly different. Three options are recommended: HR-HPV DNA testing, repeat cytology and referral for colposcopy. Reflex HR-HPV testing is the preferred option when liquid based cytology is used. European guidelines recommended that clinicians should be guided by their own National guidelines. HPV testing results determine the follow up management of women. It is necessary to use clinically validated HPV 
test and to make a distinction between HPV test performances for outcome measure precancerous disease vs outcome measure HPV presence. HC2 RLU/CO values seem to be a prognostic factor for detection of precancerous lesions of ASCUS patients. A significant decrease of positivity for HR-HPV was detected after the age of 40 years $(28 \%)$. We assume that this age will be crucial for the development of precancerous lesions within the Slovak population and thus the most suitable for HPV triage of ASCUS. As a refinement of that type of ASCUS triage we also recommend to add to the algorithm quantitative measurement of viral load in the specimens in the form of RLU/CO ratio. We assume that much lower prevalence of underlying CIN 2/CIN 3 in patients with low RLU/CO ratio (i.e. low viral load) may justify modification of the management of ASCUS in this group of women. Our statements need further studies and follow-up of much more patients.

According to our bibliographic research, this study is the first analysis of prevalence of HR-HPV of patients with cytological diagnosis of ASCUS and also the first attempt to introduce the viral load of HPV in the specimen in the management guidelines of precancerous lesions in Slovakia.

\section{References}

1. Ondrusova M, Zubor $\mathbf{P}$, Ondrus D. Time trends in cervical cancer epidemiology in the Slovakia: reflection on the non-implementation of screening with international comparisons. Neoplasma 2010; 59 (2): 121-128.

2. Safaei-Diba C, Plesko I, Hlava P(Eds). Cancer incidence in the Slovakia 2007. Bratislava: National Cancer Registry of the Slovakia, National Health Information Center, 2012.

3. Zur Hausen H. Papillomaviruses causing cancer: evasion from host-cell control in early events in carcinogenesis. J Natl Cancer Inst 2000; 92 (9): 690-698.

4. Darragh TM, Colgan TJ, Cox JT et al. The Lower Anogenital Squamous Terminology Standardization Project for HPV-Associated Lesions: background and consensus recommendations from the College of American Pathologists and the American Society for Colposcopy and Cervical Pathology. Arch Pathol Lab Med 2012; 136 (10): 1266-1297.

5. Stanko P, Kruzliak P, Labas P. Role of human papilloma virus infection and oral-genital contact in oral cancer ethiopathogenesis. Bratisl Lek Listy. 2013; 114 (6): 345-348.

6. Ho GY, Bierman R, Beardsley L. Natural history of cervicovaginal papillomavirus infection in young woman. N Engl J Med 1998; 338 (7): 423-428.

7. Liu S, Leung R, Chan K, Cheung A, Ngan H. Evaulation of a newly developed GenoArray human papillomavirus (HPV) genotyping assay and comparison with the Roche Linear Array HPV genotyping assay. J Clin Microbiol 2010; 48 (3): 758-764.

8. Wright TC Jr, Cox JT, Massad LS, Carlsson J, Twiggs LB, Wilkinson EJ; American Society for Colposcopy and Cervical Pathology. 2001 consensus guidelines for the management of women with cervical intraepithelial neoplasia. Am J Obstet Gynecol 2003; 189 (6): 295-304.

9. Mikulova J, Halanova M, Cislakova L, Pohorencova A, Szovenyiova Z, Fandakova I, Huljakova M, Klein D, Valko J, Jarcuska P. Urogenital Chlamydiosis among Slovak women. Bratisl Lek Listy. 2013; 114 (7): 398-401.

10. ASCUS-LSIL Triage Study (ALTS) Group. Results of a randomized trial on the management of cytology interpretation of atypical squamous cells of undeterminated significance. Am J Obstet Gynecol 2003; 188 (6): 1383-1392.
11. Origoni M, Carminati G, Rolla S, Clementi M, Sideri M, Sandri MT, Candiani M. Human papillomavirus viral load expressed as relative light units (RLU) correlates with the presence and grade of preneoplastic lesions of the uterine cervix in atypical squamous cells of undetermined significance (ASCUS) cytology. Eur J Clin Microbiol Infect Dis 2012; 31 (9): 2401-2406.

12. Origoni M, Carminati G, Sideri M, Clementi M, Rolla S, Candiani M. „Low grade positivity“ of HPV viral load after atypical squamous cells of undetermined significance (ASCUS) cytology identifies women at low-risk for cervical intraepithelial neoplasia grade 2 and 3. Eur J Gynecol Oncol 2012; 33 (3): 261-264.

13. Emerson RE, Puzanov A, Brunnemer C, Younger C, Cramer H. Long-term follow-up of women with atypical squamous cells of undetermined significance (ASCUS). Diagn Cytopathol 2002; 27 (3): 153-157.

14. Wright TC, Ronnett B, Kurman RJ, Ferenczy A. Precancerous lesions of the cervix. 193-252. In: Kurman RJ, Ellenson LH, Ronnett BM (Eds). Blaustein's pathology of the female genital tract, 6th ed, Philadelphia: Springer Science+ Business Media, 2011.

15. Dunne EF, Unger ER, Sternberg M, McQuillan G, Swan DC, Patel SS, Markowitz LE. Prevalence of HPV infection among females in the United States. JAMA 2007; 297 (8): 813-819.

16. Schiffman M, Solomon D. Clinical practice. Cervical-cancer screening with human papillomavirus and cytologic cotesting. N Engl J Med 2013; 369 (24): 2324-2331.

17. Snijders PJ, van den Brule AJ, Meijer CJ. The clinical relevance of human papillomavirus testing: relationship between analytical and clinical sensitivity. J Pathol 2003; 201 (1): 1-6.

18. Snijders PJ, Heideman DA, Meijer CJ. Methods for HPV detection in exfoliated cell and tissue specimens. APMIS 2010; 118 (6-7): 520-528.

19. Snijders PJ, Steenbergen RD, Heideman DA, Meijer CJ. HPV-mediated cervical carcinogenesis: concepts and clinical implications. J Pathol 2006; 208 (2): 152-164.

20. Kolníková G, Kolník P. Význam laboratórnej diagnostiky ludských papilomavírusov. Onkológia (Bratisl) 2008; 3 (4): 233-236.

21. Laudadio J. Human papillomavirus detectin: Testing methodologies and their clinical utility in cervical cancer screening, Adv Anat Pathol 2013; 20 (3): 158-167.

22. Safaeian M, Solomon D, Wacholder S, Schiffman M, Castle P. Risk of precancer and follow-up management strategies for women with human papillomavirus-negative atypical squamous cells of undetermined significance, Obstet Gynecol 2007; 109 (6): 1325-1331.

23. Cox T, Cuzick J. HPV DNA testing in cervical cancer screening: from evidence to policies. Gynecol Oncol 2006; 103 (1): 8-11.

24. Massad LS, Einstein MH, Huh WK et al. 2012 updated consensus guidelines for the management of abnormal cervical cancer screening tests and cancer precursors. J Low Genit Tract Dis 2013; 17 (5 Suppl 1): S1-S27.

25. Jordan J, Martin-Hirsh P, Arbyn M et al. Management options of case of ASCUS. 206. In: Arbyn M, Antilla A, Jordan J et al (Eds). European guidelines for quality assurance a cervical screening ( $2^{\text {nd }}$ edition). Lyon: IARC, European Communities, 2008.

26. Rowe LR, Aldeen W, Bentz JS. Prevalence and typing of HPV DNA by hybrid capture II in women with ASCUS, ASC-H, LSIL, and AGC on ThinPrep PAP tests. Diagn Cytopathol 2004; 30 (6): 426-432.

27. Jarboe EA, Venkat P, Hirsch MS, Cibas ES, Crum CP, Garner EI. A weakly positive human papillomavirus Hybrid Capture II result correlates with a significantly lower risk of cervical intraepithelial neoplasia 2,3 after atypical squamous cells of undetermined significance cytology. J Low Gen Tract Dis 2010; 14 (3): 174-178. 